\title{
BMJ Open Exploring failure of antimicrobial prophylaxis and pre-emptive therapy for transplant recipients: a systematic review
}

\author{
Anne-Grete Märtson (D , ${ }^{1}$ Martijn Bakker, ${ }^{2}$ Hans Blokzijl, ${ }^{3}$ Erik A M Verschuuren, ${ }^{4}$ \\ Stefan P Berger, ${ }^{5}$ Lambert F R Span, ${ }^{2}$ Tjip S van der Werf, ${ }^{4,5}$ \\ Jan-Willem C Alffenaar (D) ${ }^{1,6}$
}

To cite: Märtson A-G, Bakker M, Blokzijl $\mathrm{H}$, et al. Exploring failure of antimicrobial prophylaxis and pre-emptive therapy for transplant recipients: a systematic review. BMJ Open 2020;10:e034940. doi:10.1136/ bmjopen-2019-034940

- Prepublication history and additional material for this paper are available online. To view these files, please visit the journal online (http://dx.doi. org/10.1136/bmjopen-2019034940).

Received 14 October 2019 Revised 29 November 2019 Accepted 05 December 2019

Check for updates

(C) Author(s) (or their employer(s)) 2020. Re-use permitted under CC BY. Published by BMJ.

For numbered affiliations see end of article.

Correspondence to Jan-Willem C Alffenaar; j.w.c.alffenaar@umcg.nl

\section{ABSTRACT}

Objectives Infections remain a threat for solid organ and stem cell transplant recipients. Antimicrobial prophylaxis and pre-emptive therapy have improved survival of these patients; however, the failure rates of prophylaxis are not negligible. The aim of this systematic review is to explore the reasons behind failure of antimicrobial prophylaxis and pre-emptive therapy.

Setting This systematic review included prospective randomised controlled trials and prospective single-arm studies.

Participants The studies included were on prophylaxis and pre-emptive therapy of opportunistic infections in transplant recipients. Studies were included from databases MEDLINE, CENTRAL and Embase published unti October first 2018.

Primary and secondary outcome measures Primary outcome measures were breakthrough infections, adverse events leading to stopping of treatment, switching medication or dose reduction. Secondary outcome measures were acquired resistance to antimicrobials, antifungals or antivirals and death.

Results From 3317 identified records, 30 records from 24 studies with 2851 patients were included in the systematic review. Seventeen focused on prophylactic and pre-emptive treatment of cytomegalovirus and seven studies on invasive fungal infection. The main reasons for failure of prophylaxis and pre-emptive therapy were adverse events and breakthrough infections, which were described in $54 \%$ (13 studies) and 38\% (9 studies) of the included studies, respectively. In $25 \%$, six of the studies, a detailed description of patients who experienced failure of prophylaxis or pre-emptive therapy was unclear or lacking.

Conclusions Our results show that although failure is reported in the studies, the level of detail prohibits a detailed analysis of failure of prophylaxis and preemptive therapy. Clearly reporting on patients with a negative outcome should be improved. We have provided guidance on how to detect failure early in a clinical setting in accordance to the results from this systematic review.

PROSPERO registration number CRD42017077606.
Strengths and limitations of this study

- To reduce selection bias, all the studies were independently reviewed and risk of bias was assessed by two authors and disagreements solved by including a third reviewer.

- One limitation of this systematic review is that the included studies were recruiting only adult patients.

- Inclusion of single-arm studies could be a potential limitation as these can cause bias in the systematic review.

- To reach a broad scope for the systematic review three databases, MEDLINE, Embase and CENTRAL, were searched.

- The systematic review was reported according to Preferred Reporting Items for Systematic Reviews and Meta-Analyses guidelines.

\section{INTRODUCTION}

In spite of novel immunosuppressive regimens and antimicrobial prophylaxis, infectious complications remain a threat for solid organ and stem cell transplant (SCT) recipients. ${ }^{1-5}$ These patients are especially susceptible to opportunistic infections like cytomegalovirus (CMV), Pneumocystis jirovecii pneumonia (PCP), febrile neutropoenia, human herpesvirus 6 (HHV-6) and invasive fungal infections (IFI) ${ }^{1-5}$ Graft failure is a major risk of these opportunistic infections. ${ }^{6-8}$ In recent years, organ transplantation and immunosuppressive regimes have developed greatly and thus become available for a wider patient population. This requires adequate antimicrobial prophylaxis guidelines and studies supporting the scientific evidence.

Antimicrobial prophylaxis and pre-emptive therapy are used as preventive measures; however, these vary notably among different transplant centres. ${ }^{910}$ This can be explained by differences in local setting and lack of 
3317 records identified through database search

595 from PubMed/MEDLINE

1998 from Embase

724 from CENTRAL

603 duplicate records removed

3317 records examined

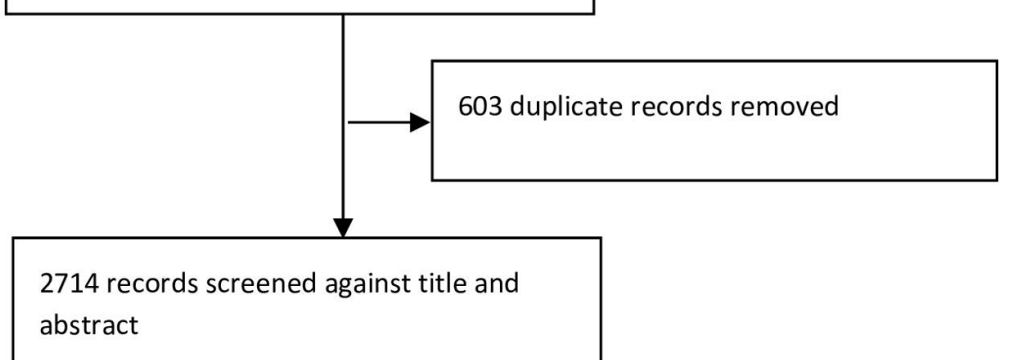

2543 records excluded because they did not met the inclusion criteria

171 full-text articles screened matching inclusion criteria

142 records excluded

86 conference proceedings

25 study design not applicable

15 unsuitable patient cohort

7 unsuitable intervention

4 editorials/letter to the editors

3 study protocols

1 study published in Japanese

1 record identified through searching references of full texts

n

24 studies (in 30 articles) included in the systematic review

Figure 1 PRISMA diagram. PRISMA, Preferred Reporting Items for Systematic Reviews and Meta-Analyses.+++ 


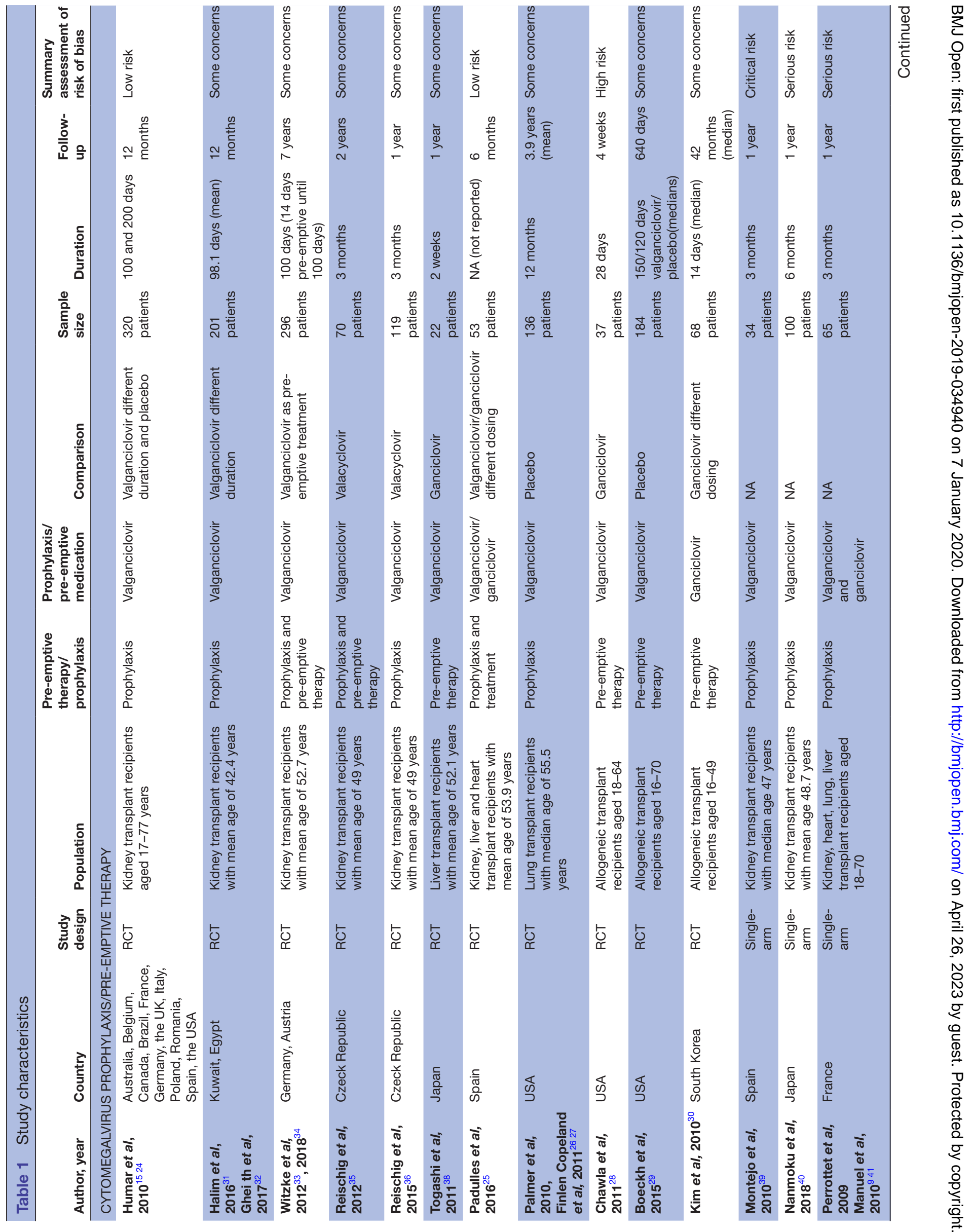




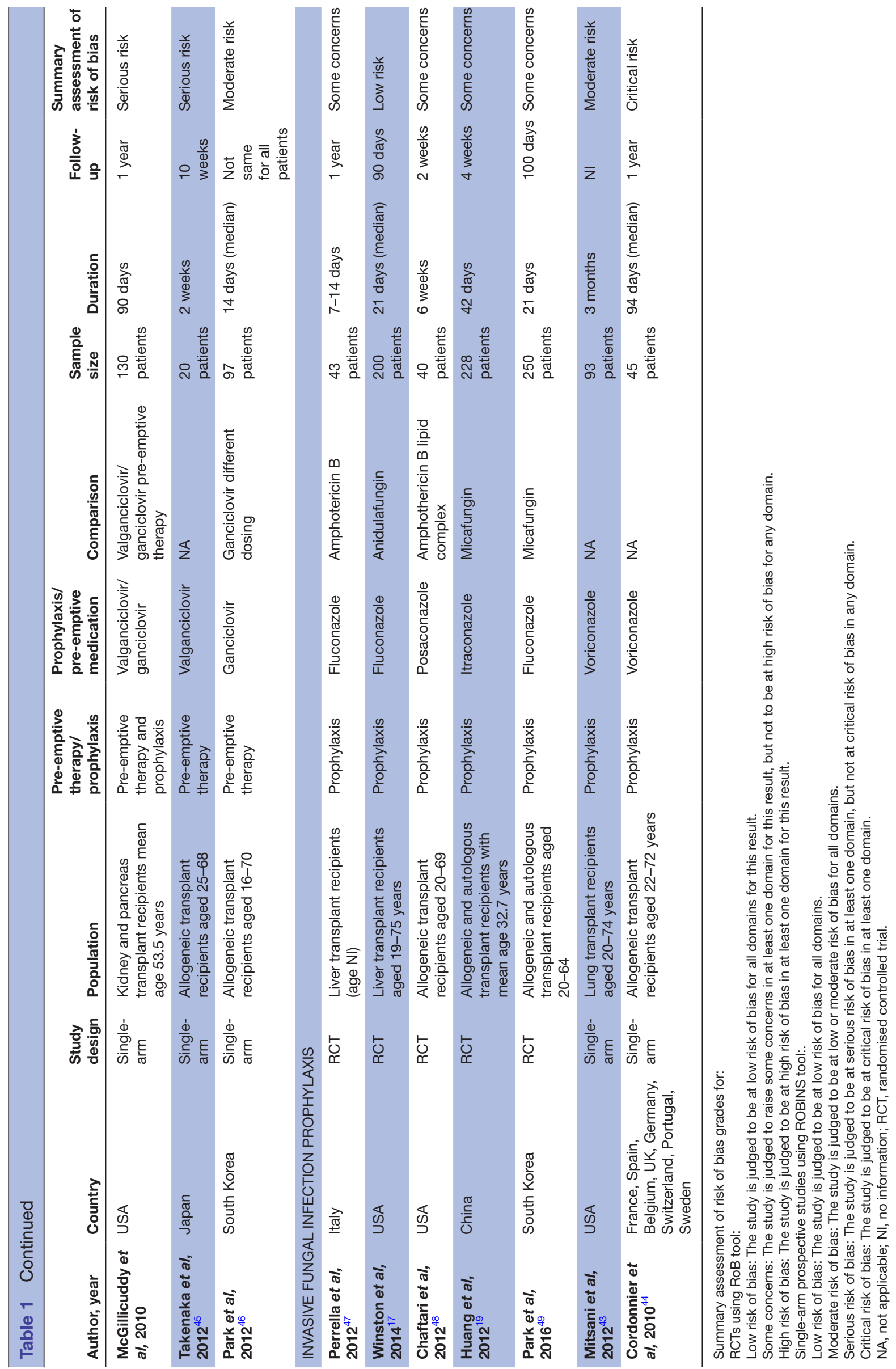


high level evidence in antifungal, antimicrobial and antiviral prophylaxis guidelines and fairly dated evidence for some infections like PCP and febrile neutropenia. ${ }^{5}$ 11-14 Although prophylaxis has proven to be a beneficial strategy, breakthrough infections and adverse events resulting in discontinuation of prophylaxis occur. ${ }^{15-17}$ The rates of failure of prophylaxis have remained around $10 \%-20 \%$ for opportunistic infections. ${ }^{15}{ }^{18-20}$ In order to be able to optimise prophylaxis, it is important to understand underlying causes for failure. This systematic review will identify (1) causes of failure of prophylactic treatment, (2) factors that might contribute to failure of prophylaxis and (3) different approaches for administering prophylaxis.

This systematic review aimed to summarise the main reasons why prophylaxis and pre-emptive therapy has failed in solid organ and allogeneic SCT recipients and how failure is reported in prospective studies. In this review, we focus on failure of prophylactic therapy, during the treatment window.

\section{METHODS}

\section{Definition of failure}

In this systematic review, we have defined failure of prophylaxis and pre-emptive therapy as stopping or changing the therapy during the study period for any stated reason. For example, failure could be a breakthrough infection, nonadherence, adverse events leading to stopping of therapy, etc. Even if some of the side effects could be anticipated, if this leads to stopping of therapy, it was defined as failure. We did not look into infections in the postprophylactic period.

\section{Search strategy and selection criteria}

Methods of the analysis and inclusion criteria were specified in advance and documented in a protocol, which is available online: https://www.crd.york.ac.uk/prospero/display_record.php?RecordID $=77606 \quad$ (online supplementary appendix 1). This report follows the Preferred Reporting Items for Systematic Reviews and Meta-Analyses (PRISMA) checklist (online supplementary appendix 2). ${ }^{21}$ This systematic review included prospective randomised controlled trials (RCT) and prospective single-arm studies from 1 January 2010 to 1 October 2018. The starting date was 1 January 2010 due to the changes in management of different infections in recent decades and to include the most recent evidence. ${ }^{11} 12$ There were no limitations for the patient setting, publication status and language. We analysed the failure during prophylaxis and pre-emptive therapy, thus the follow-up time varied. The review included patients (16 years and older) who had received either allogeneic stem cell, lung, kidney, liver, heart, pancreas or small bowel transplantation. Studies done on children under 16 years were not included as this would have introduced more variability and require a separate analysis, thus were out with the scope of this review. Moreover, these patients were receiving trimethoprim/sulfamethoxazole for prophylaxis of PCP, ciprofloxacin for prophylaxis of febrile neutropenia, ganciclovir and/or valganciclovir for prophylaxis or pre-emptive-therapy of CMV and human herpesvirus 6 (HHV-6); or posaconazole or voriconazole or fluconazole or itraconazole for prophylaxis of invasive fungal infections (IFI). Primary outcome measures were breakthrough infections, adverse events leading to stopping of treatment, switching medication or dose reduction. Secondary outcome measures were acquired resistance to antimicrobials, antifungals or antivirals and death.

To identify studies for this systematic review, the following databases were searched: MEDLINE (PubMed), EMBASE and Cochrane Central Register of Controlled Trials (CENTRAL). The search was performed on 1 October 2018. The search strategy included MeSH terms and variations of transplant types, medications and prophylaxis ('Antibiotic Prophylaxis' (Mesh) OR antimicrobial OR antimicrobial) AND (Host OR Transplants OR Transplantation OR Transplant Recipients OR immunocompromised OR transplant* OR kidney transplant*). The full-search strategies of all databases can be found in online supplementary appendix 3 . The searches were done by AGM and MB. For screening, we used Covidence software (www.covidence.org).

The literature search and data extraction for inclusion and eligibility for this systematic review were done according to the inclusion criteria by AGM and MB independently. If there were discrepancies between the results, these were discussed and resolved with JWA or with consensus. If there was data missing or additional questions from the selected studies, then the authors of these studies were contacted. We excluded conference proceedings, retrospective studies, reviews, editorials and letters to the editor.

\section{Outcomes, data extraction and quality}

Data were extracted by AGM and independently checked by MB. Disagreement between reviewers was resolved by discussion with a third reviewer JWA. Data were extracted from each included trial on: characteristics of patients, type of intervention, study design, study population, outcome measurement, reasons for failure of prophylaxis or pre-emptive therapy (stopping of prophylaxis/preemptive therapy), main conclusions by authors, strengths and limitations (online supplementary appendix 4).

We considered performing a meta-analysis for our systematic review, however because the patient cohorts include different transplantations and varied interventions, the studies were too heterogeneous, we decided to do a qualitative systematic review. The studies were divided into CMV and IFI prophylaxis and pre-emptive treatment groups.

Risk of bias in individual studies was assessed independently by AGM and MB. For assessing bias in individual studies, Revised Cochrane risk-of-bias tool for randomised trials (RoB 2) was used for RCTs and Risk of Bias in 
Non-randomised Studies of Interventions (ROBINS-I) was used for prospective single-arm studies. ${ }^{22} 23$

\section{RESULTS}

The search identified 3317 records for inclusion in the review. In total, 603 duplicate records were removed, and 2543 records were excluded after screening of title and abstract. Full-text screening of 171 articles resulted in 24 studies (in 30 articles) (PRISMA flow diagram in figure 1) to be included in the final review. We decided to exclude one full text in Japanese as it would not likely change the outcome of our systematic review. We contacted corresponding authors of 24 studies ( 30 articles) for further information about reasons for preliminary stopping of prophylaxis and overall adherence to treatment, seven responded and three of those sent prespecified protocols; however, none provided additional information about failure of prophylaxis or pre-emptive treatment. The individual study characteristics and risk of bias are presented in table 1.

We identified 24 studies including 2851 patients. Seventeen studies (11 RCTs ${ }^{1524-38}$ and six single-arm ${ }^{39-46}$ ) with 1952 subjects focused on CMV prophylaxis and seven studies (5 RCTs ${ }^{17} 19$ 47-49 and 2 single-arm ${ }^{43}{ }^{44}$ ) with 899 subjects focused on IFI prophylaxis.

Of the 17 CMV studies, eight included only valganciclovir, two only ganciclovir, five included both valganciclovir and ganciclovir, two valaciclovir and valganciclovir. From all 17 CMV studies, seven focused only on prophylaxis, six only on pre-emptive therapy, three both on prophylaxis and pre-emptive therapy and one on prophylaxis and therapy.

The IFI studies varied with regard to study medication and patient group. Three studies evaluated fluconazole (comparison amphotericin $\mathrm{B}$, anidulafungin and micafungin), one posaconazole (comparison amphotericin B lipid complex), one itraconazole (comparison micafungin) and two single-arm studies had voriconazole as study medication.

\section{Failure of prophylaxis and pre-emptive therapy}

No specific information about failure during prophylaxis or pre-emptive therapy was given in $25 \%$ (six studies: four being RCTs) of the included studies. ${ }^{19} 252840424750$ Four of these studies did record follow-up infections or long-term failure of prophylaxis therapy after cessation of prophylaxis $^{19404247}$ and for one RCT the time-point was not specified. ${ }^{46}$

The most common reasons for failure of CMV prophylaxis (1524 study subjects) and CMV pre-emptive therapy (428 study subjects) were adverse events ${ }^{1525} 272930364246$ and breakthrough CMV. ${ }^{15} 29414246$ For IFI prophylaxis (899 study subjects), it was adverse events ${ }^{19} 4344849$ and IFI. ${ }^{17194449}$ Overall, the adverse events and breakthrough infections were described in 54\% (13 studies) and 38\% (9 studies) of the studies respectively. In table 2, the reasons for stopping prophylaxis are described in detail.
The detailed information about failure in the CMV (1506 study subjects) and IFI (761 study subjects) RCT groups are summarised in figures 2 and 3 .

From the secondary outcomes, death was reported more frequently-in $33 \% \quad(n=8)$ of the studies, death was the reason for failure. ${ }^{172730} 3135364446$ The secondary outcome resistance to antimicrobials, antivirals and antifungals was addressed in the introductions and discussions of the included studies, however not regarded as failure of therapy. Moreover, the presence and/or measurement of resistance to the study drug was described in two studies. ${ }^{2729}$ Boeckh et al report no resistance genes in the investigated patients and Palmer et al report one patient with known resistance to ganciclovir.

Not all identified cases of failure could be clarified even after contact with the authors of the studies. The reasons for stopping prophylaxis and pre-emptive therapy, patient reasons or physician or sponsor decision, ${ }^{2729}$ were not explained in any of these studies. Moreover, in two studies, ${ }^{24} 27$ it was stated that prophylaxis was stopped because of other reasons. Adverse events, breakthrough infections and cause of death were mostly described in further detail in the included studies. ${ }^{15-17} 27$ 29-31 35 42-44 464849 However, reasons 'other' ${ }^{1527}$ physician/investigator decision ${ }^{17272949}$ and patients discretion ${ }^{15}$ were grouped together in studies and not described in detail.

\section{Risk of bias across studies}

Risk of bias was assessed using five domains for RCTs: randomisation, assignment and adherence to intervention, measurement of outcome, missing data and selection of the reported results. For single-arm/observational studies domains were used: confounding, selection of participants, classification of interventions, missing data, measurement of outcomes and selection of the reported result. We concluded that all 16 RCTs and six single-arm had low risk of bias, three had some concerns and two had high risk of bias. Risk of bias in RCTs and single-arm studies is presented in tables 3 and 4 .

\section{DISCUSSION}

We aimed to explore the reasons why prophylaxis and preemptive therapy failed in transplant recipients. Twentyfour studies were included into this systematic review. We concluded that the main reason for stopping prophylaxis was adverse events for both CMV and IFI prophylaxis and CMV pre-emptive therapy. We did not observe notable differences between the prophylaxis and pre-emptive therapy groups. This result was expected as cessation of ganciclovir therapy is often described due to debilitating side-effects, especially bone marrow suppression, ${ }^{15}$ which is even more problematic in haematological patients. ${ }^{28}$ Different antifungal agents have a diverse safety profile. Our results were also in line with the common side-effects of antifungals as we observed nausea and vomiting in azoles and nephrotoxicity in Amphotericin B as reasons for discontinuation of therapy. ${ }^{43} 46$ 


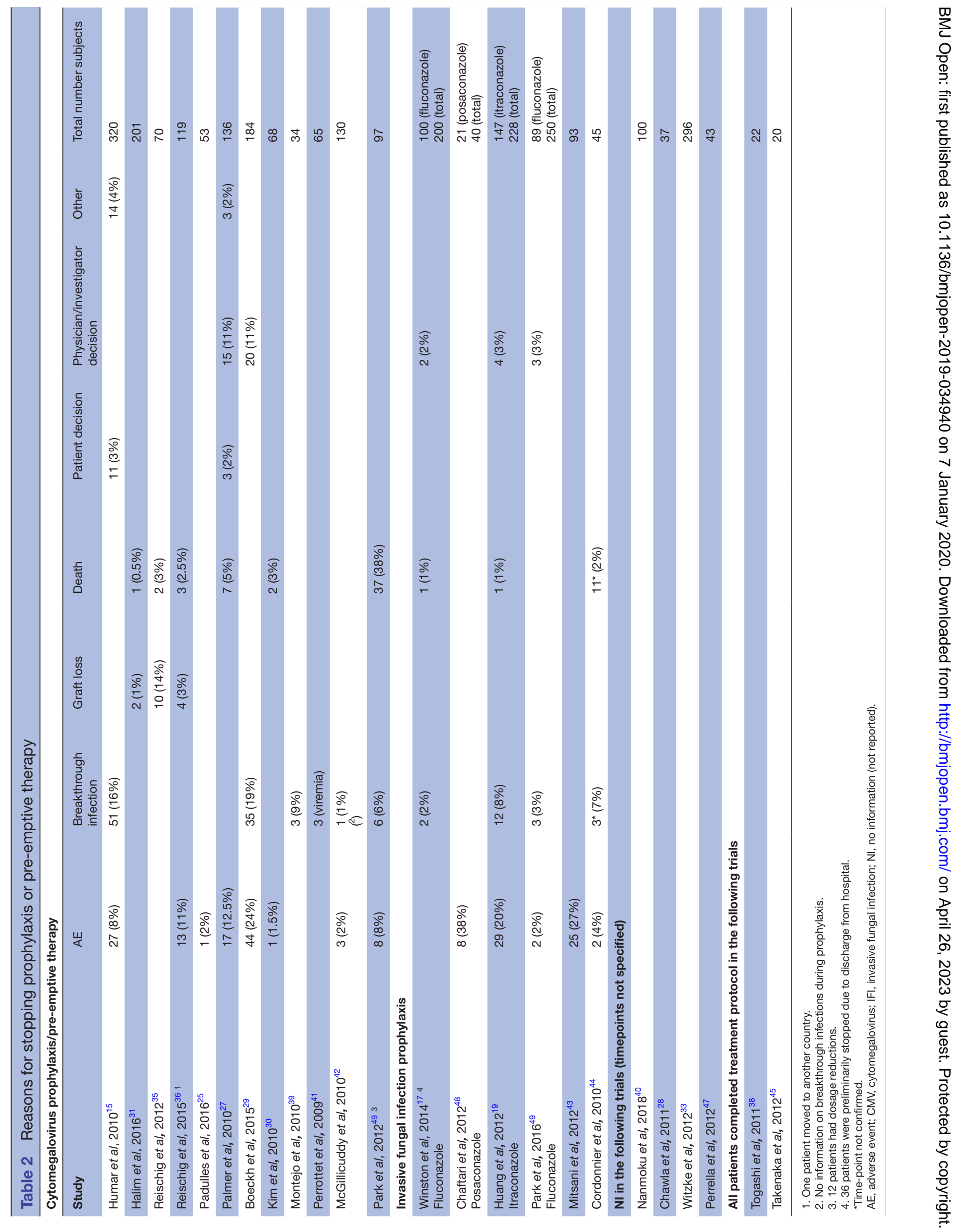




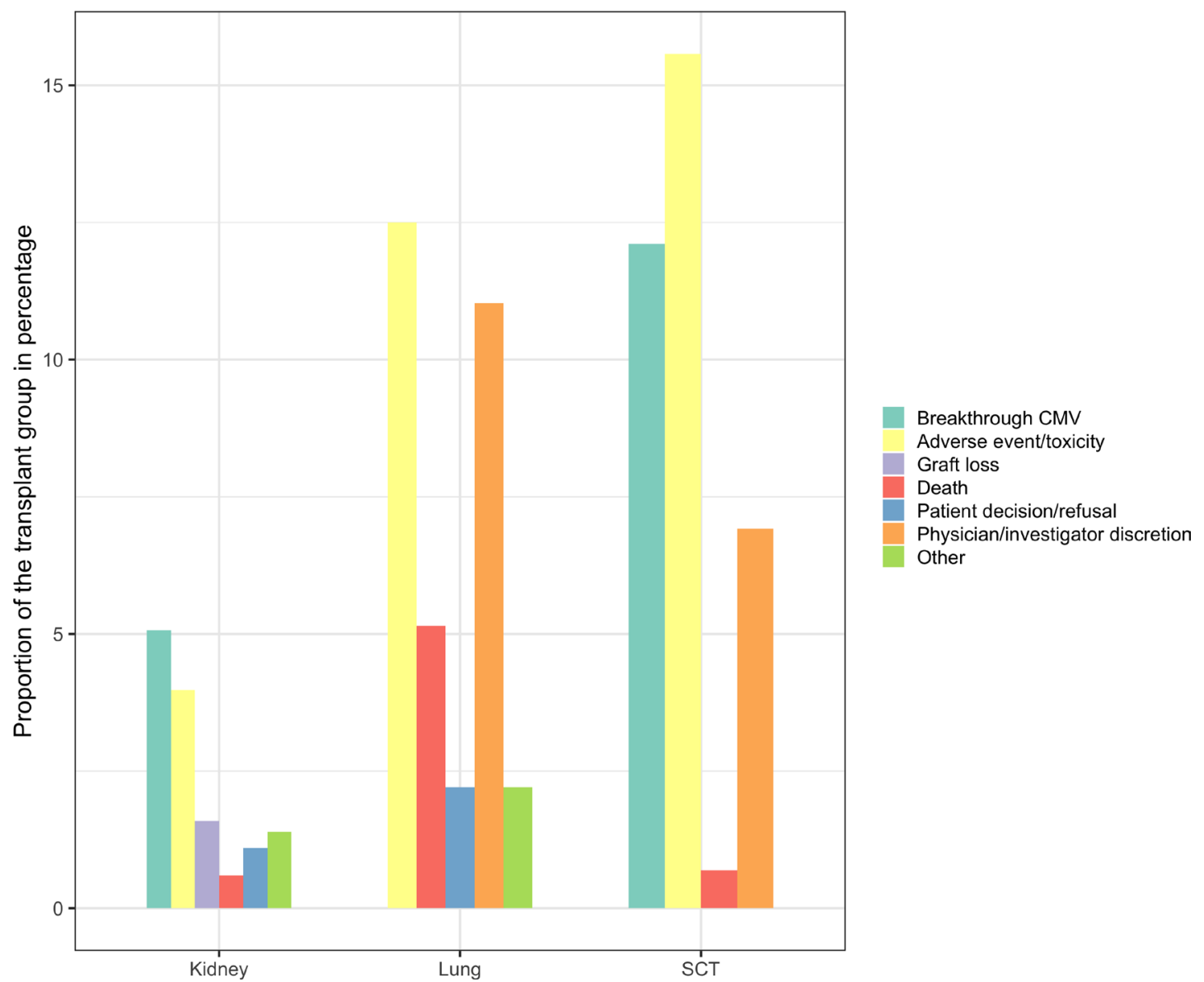

Figure 2 Failure of CMV prophylaxis and pre-emptive therapy in $11 \mathrm{RCTs}$ (y-axis represents the proportion of the transplant group in percentages and $\mathrm{x}$-axis represents transplant). CMV, cytomegalovirus; RCTs, randomised controlled trials.

Adverse events are known to be under-reported so the numbers that we summarised in this systematic review might be underestimated. ${ }^{51}{ }^{52} \mathrm{~A}$ review by Golder $e t a \tilde{l}^{2}$ looked into different study designs and reporting of adverse events. The authors outlined that there is wide under-reporting of adverse events across different study designs and even more in the unpublished data. Unfortunately, contacting the authors of the studies included in this systematic review did not result in additional information about the reported adverse events. Meta-analysis was not done as the studies were too heterogeneous.

Surprisingly, reporting of failure was insufficient in $25 \%$ of RCTs and $50 \%$ of prospective single-arm studies. One-fourth of the studies did not report on preliminarily cessation of prophylaxis nor underlying reason. More worrisome was the fact that discontinuation of prophylaxis was mostly explained as adverse events and breakthrough infections, while patient and physicians' discretion as reasons were grouped together. On the other hand, we observed that only five studies did record infections that occurred after the prophylaxis had been stopped. This is concerning, as in a clinical setting and in developing guidelines, it is important to make a distinction of breakthrough infections during and after prophylaxis and pre-emptive therapy. ${ }^{53}$ Also, it makes it more difficult to compare the efficacy of different medications, for example when using ganciclovir and letermovir. ${ }^{53}$ Furthermore, one of the included $\mathrm{RCTs}^{17}$ reported early discharge from hospital as the main argument for stopping of prophylaxis. In addition, some reasons were not clarified, for example physician and patient discretion were combined in two of the included studies. A systematic review published in 2018 explored the efficacy and safety of CMV prophylaxis; adverse events and breakthrough infections were addressed, however the authors did not explicitly report additional information why patients stopped within these studies. ${ }^{54}$ Similarly, in a PCP prophylaxis systematic review, the authors described adverse events as reason for discontinuation while not mentioning adherence, resistance or patients' choice. ${ }^{14}$

A systematic review about quality of reporting RCTs in medical oncology described that $79 \%$ of the adverse events in studies are reported according to Consolidated Standards of Reporting Trials (CONSORT) criteria, although the description of participants and preliminary stopping in each stage of the study was done correctly in only $59 \%$ of the studies. ${ }^{55}$ It has been argued that perhaps, poor reporting is deliberate to mask the shortcomings in study design. ${ }^{556}$ One may expect that in a prospective study, the patients are recruited and analysed prospectively thus the data about failure of prophylaxis like adverse events should be readily obtainable. Thus, registering 


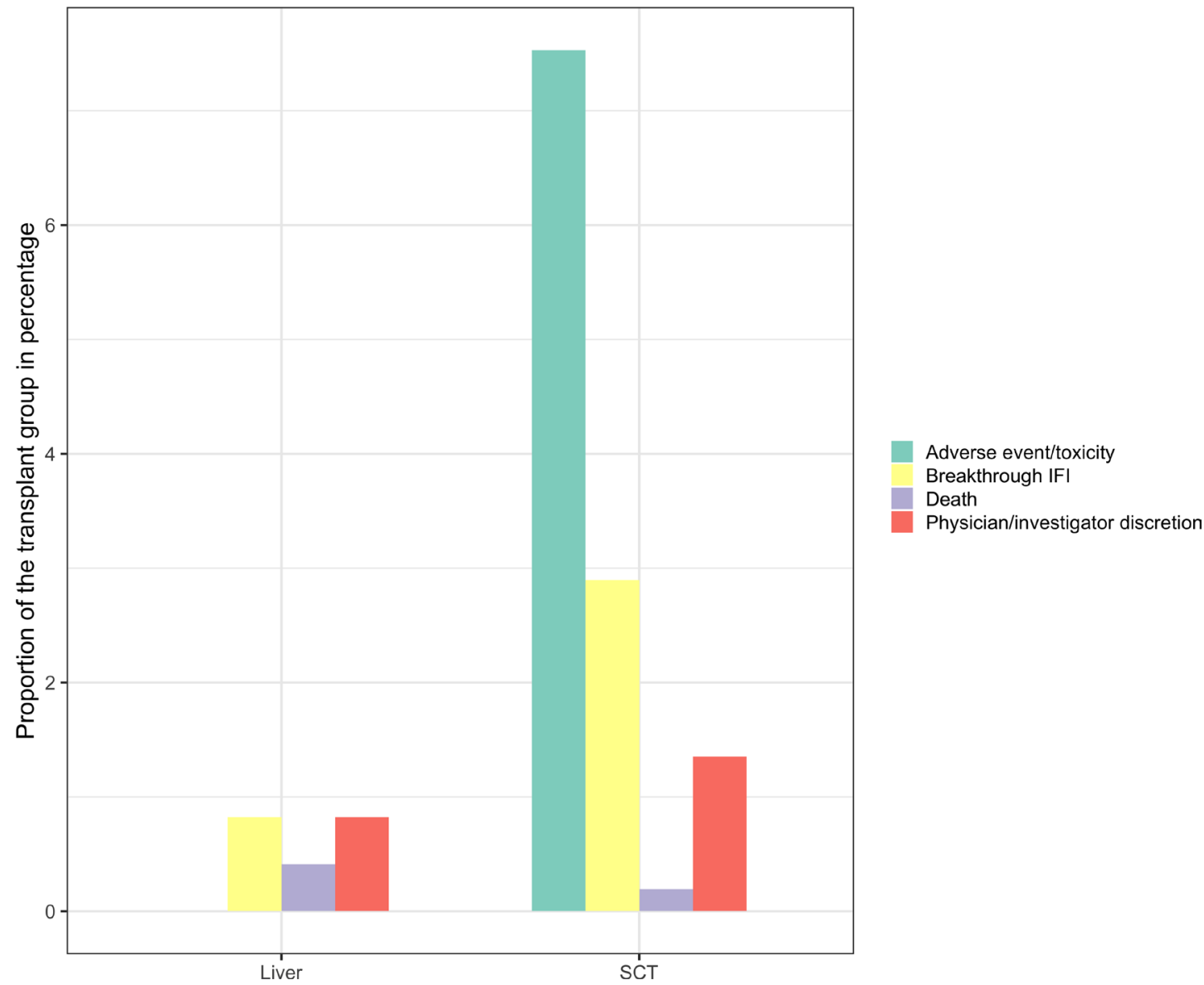

Figure 3 Failure of IFI prophylaxis in five RCTs (y-axis represents the proportion of the transplant group in percentages and $\mathrm{x}$-axis represents transplant). For one study, $15 \%$ of failure was caused by patients discharged early, this has not been included in this figure. IFI, invasive fungal infection.

study protocols in clinical trial registers and following reporting guidelines helps consistent and more straightforward reporting of results. ${ }^{57} 58$ Goldacre and colleagues have looked into reporting of clinical trials specifically with regard to outcomes. They concluded that most of the studies did not have sufficient reporting-either outcomes were added or prespecified outcomes were not reported. ${ }^{59}$ These results raise concern to also whether failure is reported as per protocol. In our case, we faced a substantial quantity of missing data on failure and it is not always clear to us how studies were conducted with respect to exclusion of patients. An additional statement to the CONSORT criteria regarding better reporting of harms in randomised trials is a useful guidance document to improve conduct of studies and address shortcomings. ${ }^{60}$

According to our search criteria, we also planned to include studies looking into prophylaxis and pre-emptive therapy of PCP, HHV-6 virus and febrile neutropenia. Surprisingly, we were not able to identify suitable studies looking into prophylaxis of these infections for our systematic review. Current guidelines for the treatment of these infections use case reports, retrospective studies, surveillance studies and outdated literature to give recommendations. ${ }^{56162}$ For instance, a systematic review focusing on PCP prophylaxis in non-HIV immunocompromised patients is used as main guidance of prophylaxis in this patient group; the studies included in this review date back from 1974 to 2008 (13 in the range 1977-1990). ${ }^{14}$ Moreover, the prophylaxis of febrile neutropenia is widely supported by a systematic review that included studies with quinolones published from 1980 to 2010 (10 in the range 1980-1997) ${ }^{13}$ Half of the studies in these reviews were published more than 20 years ago. Certainly, the evidence from these reviews are relevant to the field; however, the landscape of treatment of transplant recipients has changed-notably, with the emergence of resistant pathogens ${ }^{63-66}$ and new data in today's setting is needed to aid the update of clinical guidelines. ${ }^{62}$

As mentioned before, detailed data about failure of prophylaxis and pre-emptive therapy were lacking in some studies and no additional information was obtained when reaching out to the authors. Furthermore, a limitation of our study was the restriction of start date (1 January 2010). This was done to avoid the effect of the significant change in management of infections and focus on the most recent evidence. ${ }^{11} 12$ Another limitation is that we did not include studies about children. On the other hand, included studies already showed a heterogeneous variety of patient populations (eg, autologous and allogeneic SCT recipients), ${ }^{19} 48$ various types of infections and 
Table 3 Risk of bias in RCTs using RoB too

\begin{tabular}{|c|c|c|c|c|c|c|}
\hline & $\begin{array}{l}\text { Randomisation } \\
\text { process }\end{array}$ & $\begin{array}{l}\text { Assignment of } \\
\text { intervention }\end{array}$ & $\begin{array}{l}\text { Adhering to } \\
\text { intervention }\end{array}$ & $\begin{array}{l}\text { Measurement of } \\
\text { outcomes }\end{array}$ & $\begin{array}{l}\text { Missing } \\
\text { outcome data }\end{array}$ & $\begin{array}{l}\text { Selection of the } \\
\text { reported result }\end{array}$ \\
\hline Humar et al, $2010^{15}$ & + & + & + & + & + & $?$ \\
\hline Halim et al, $2016^{31}$ & $?$ & + & + & $?$ & + & $?$ \\
\hline Witzke et al, $2012^{33}$ & + & + & + & $?$ & + & $?$ \\
\hline Reischig et al, $2012^{35}$ & + & + & + & $?$ & + & $?$ \\
\hline Reischig et al, $2015^{36}$ & + & + & + & $?$ & + & $?$ \\
\hline Padulles et al, $2016^{25}$ & + & + & + & + & + & $?$ \\
\hline Palmer et al, $2010^{27}$ & + & + & $?$ & + & + & $?$ \\
\hline Chawla et al, $2011^{28}$ & $?$ & $?$ & $?$ & $?$ & + & $?$ \\
\hline Boeckh et al, $2015^{29}$ & + & + & $?$ & + & + & $?$ \\
\hline Chaftari et al, $2012^{48}$ & + & $?$ & $?$ & $?$ & + & $?$ \\
\hline Huang et al, $2012^{19}$ & + & + & $?$ & $?$ & + & $?$ \\
\hline Park et al, $2016^{49}$ & ? & + & + & ? & + & $?$ \\
\hline
\end{tabular}

+low risk; ?, some concerns.

$\mathrm{RCT}$, randomised controlled trials; RoB, risk-of-bias.

prophylaxis and pre-emptive therapy studies; therefore, we believe that adding paediatric studies would have further increased variability of our results.

There remains a variety of different practices between centres complicating patient transfers between hospitals. ${ }^{967}$ We believe that having information about the discontinuation of failure of prophylaxis and pre-emptive therapy could provide valuable information for guideline committees, medical practitioners and researchers conducting studies with these medications. Without this information, similar errors could be repeated in different studies.

There are several ways to predict failure in a clinical setting. Detecting adverse events and avoiding breakthrough infections can be done by approaching the

Table 4 Risk of bias in single-arm studies using ROBINs tool

\begin{tabular}{|c|c|c|c|c|c|c|c|}
\hline & Confounding & $\begin{array}{l}\text { Selection of } \\
\text { participants }\end{array}$ & $\begin{array}{l}\text { Classification } \\
\text { of } \\
\text { interventions }\end{array}$ & $\begin{array}{l}\text { Deviations } \\
\text { from intended } \\
\text { interventions }\end{array}$ & Missing data & $\begin{array}{l}\text { Measurement } \\
\text { of outcomes }\end{array}$ & $\begin{array}{l}\text { Selection of } \\
\text { the reported } \\
\text { result }\end{array}$ \\
\hline $\begin{array}{l}\text { Montejo et al, } \\
2010^{39}\end{array}$ & - & $?$ & + & $\mathrm{NI}$ & - & $?$ & $\mathrm{NI}$ \\
\hline $\begin{array}{l}\text { Nanmoku et al, } \\
2018^{40}\end{array}$ & * & + & + & + & + & $?$ & $\mathrm{NI}$ \\
\hline $\begin{array}{l}\text { McGillicuddy et } \\
\text { al, } 2010^{42}\end{array}$ & * & + & + & * & * & $?$ & $\mathrm{NI}$ \\
\hline $\begin{array}{l}\text { Takenaka et al, } \\
2012^{45}\end{array}$ & * & $?$ & + & $?$ & * & $?$ & $\mathrm{NI}$ \\
\hline $\begin{array}{l}\text { Park et al, } \\
2012^{46}\end{array}$ & $?$ & + & + & $?$ & + & $?$ & $\mathrm{NI}$ \\
\hline $\begin{array}{l}\text { Cordonnier et } \\
\text { al, } 2010^{44}\end{array}$ & * & * & + & $?$ & $?$ & $?$ & $\mathrm{NI}$ \\
\hline
\end{tabular}

+, low risk; ?, moderate risk; *, serious risk; -, critical risk.

$\mathrm{NI}$, no information; ROBINS, Risk of Bias in Non-randomised Studies of Interventions. 


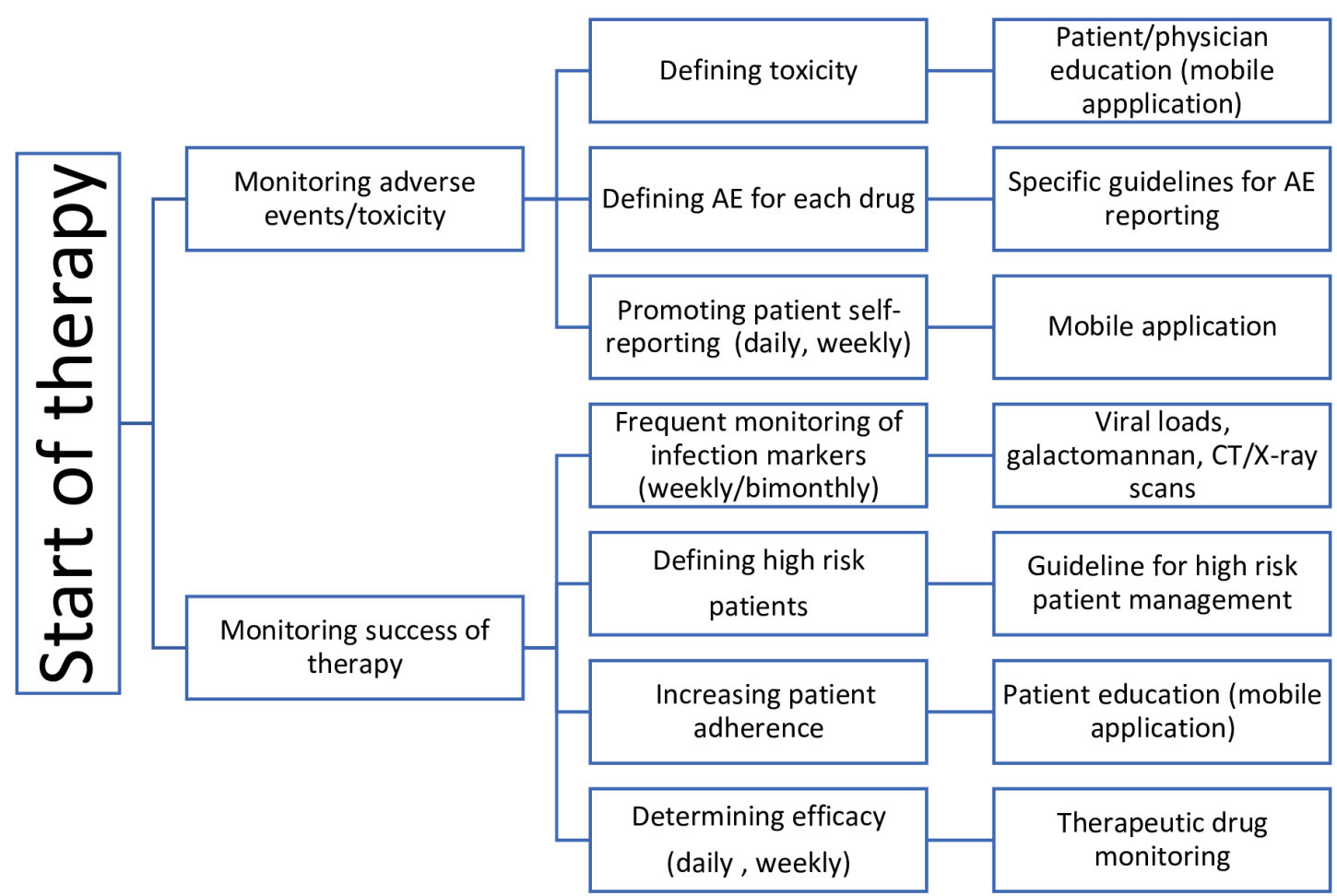

Figure 4 Detecting failure in a clinical setting. AE, adverse event.

patient or treating the physician. For example, using mobile applications for reporting adverse events, ${ }^{68}$ defining the high-risk patients ${ }^{69} 70$ and therapeutic drug monitoring of potentially toxic medications. ${ }^{71}$

In figure 4, we have summarised guidance on how to detect failure early. ${ }^{68-76}$ As mentioned above, there are multiple studies that do not report the true breakthrough infections, only postprophylaxis infections are reported. In addition, we believe that defining the potential adverse events before and describing the method of measurement of these could increase adherence. Other supporting background from the current systematic review for this figure is presented in Appendix 5.

\section{Conclusions}

In general, RCTs and prospective single-arm studies about prophylaxis and pre-emptive therapy of opportunistic infections should provide more in-depth information about failure. The main reason why prophylaxis or preemptive therapy is stopped are adverse events; however, these may well be underreported. Thus, the management and reporting of adverse events is critically important and should be improved in clinical studies. In addition, our results suggest partially biased approach in the publication of clinical studies and therefore there are insufficient data to support evidence-based decision-making in prophylaxis of PCP, HHV-6 and febrile neutropenia.

\section{Author affiliations}

${ }^{1}$ Department of Clinical Pharmacy and Pharmacology, University Medical Center Groningen, University of Groningen, Groningen, The Netherlands

${ }^{2}$ Department of Hematology, University Medical Center Groningen, University of Groningen, Groningen, The Netherlands
${ }^{3}$ Department of Gastroenterology and Hepatology, University Medical Center Groningen, University of Groningen, Groningen, The Netherlands

${ }^{4}$ Department of Pulmonary Diseases and Tuberculosis, University Medical Center Groningen, University of Groningen, Groningen, The Netherlands

${ }^{5}$ Department of Internal Medicine, University Medical Center Groningen, University of Groningen, Groningen, The Netherlands

${ }^{6}$ The University of Sydney, Sydney Pharmacy School, Sydney, New South Wales, Australia

Acknowledgements The authors would like to thank Mariska M.G. Leeflang for her suggestions and help while analyzing and compiling the data of this systematic review.

Contributors AGM did the screening, writing, data analysis, risk of bias analysis, searches and planning; MB did the screening, searches, data analysis, writing; $\mathrm{HB}$, EAMV, SPB, LFRS and TSvdW did the planning, writing, reviewing of manuscript; JWA did the writing, analysis and planning.

Funding AGM was funded by Marie Skłodowska-Curie Actions, Grant Agreement number: 713660 — PRONKJEWAIL — H2020-MSCA-COFUND-2015

Competing interests None declared.

Patient consent for publication Not required.

Provenance and peer review Not commissioned; externally peer reviewed.

Data availability statement The data that support the findings of this study are available from the corresponding author.

Open access This is an open access article distributed in accordance with the Creative Commons Attribution 4.0 Unported (CC BY 4.0) license, which permits others to copy, redistribute, remix, transform and build upon this work for any purpose, provided the original work is properly cited, a link to the licence is given, and indication of whether changes were made. See: https://creativecommons.org/licenses/by/4.0/.

ORCID iDs

Anne-Grete Märtson http://orcid.org/0000-0001-6478-1959

Jan-Willem C Alffenaar http://orcid.org/0000-0001-6703-0288

\section{REFERENCES}

1 Ullmann AJ, Schmidt-Hieber M, Bertz H, et al. Infectious diseases in allogeneic haematopoietic stem cell transplantation: 
prevention and prophylaxis strategy guidelines 2016. Ann Hematol 2016;95:1435-55.

2 Menzin J, Meyers JL, Friedman M, et al. The economic costs to United States hospitals of invasive fungal infections in transplant patients. Am J Infect Control 2011;39:e15-20.

3 Hakimi Z, Aballéa S, Ferchichi S, et al. Burden of cytomegalovirus disease in solid organ transplant recipients: a national matched cohort study in an inpatient setting. Transpl Infect Dis 2017;19.

4 Iriart X, Bouar M, Kamar N, et al. Pneumocystis pneumonia in solidorgan transplant recipients. JoF 2015;1:293-331.

5 Taplitz RA, Kennedy EB, Bow EJ, et al. Antimicrobial prophylaxis for adult patients with cancer-related immunosuppression: ASCO and IDSA clinical practice guideline update. J Clin Oncol September 2018.

6 Alsaeed M, Husain S. Infections in heart and lung transplant recipients. Crit Care Clin 2019;35:75-93.

7 Freeman, Jr. RB. The 'Indirect' Effects of Cytomegalovirus Infection. Am J Transplant 2009;9:2453-8.

8 Fishman JA. Infection in solid-organ transplant recipients. N Engl J Med 2007:357:2601-14.

9 Manuel O, Kralidis G, Mueller NJ, et al. Impact of antiviral preventive strategies on the incidence and outcomes of cytomegalovirus disease in solid organ transplant recipients. Am J Transplant 2013;13:2402-10.

10 Navarro D, San-Juan R, Manuel O, et al. Cytomegalovirus infection management in solid organ transplant recipients across European centers in the time of molecular diagnostics: an ESGICH survey. Transpl Infect Dis 2017;19.

11 Patterson TF, Thompson GR, Denning DW, et al. Practice guidelines for the diagnosis and management of aspergillosis: 2016 update by the infectious diseases Society of America. Clin Infect Dis. 2016;63:e1-60.

12 Kotton CN, Kumar D, Caliendo AM, et al. The third International consensus guidelines on the management of cytomegalovirus in solid-organ transplantation. Transplantation 2018;102:900-31.

13 Gafter-Gvili A, Fraser A, Paul M, et al. Antibiotic prophylaxis for bacterial infections in afebrile neutropenic patients following chemotherapy. Cochrane database Syst Rev 2012;27.

14 Stern A, Green H, Paul M, et al. Prophylaxis for Pneumocystis pneumonia (PCP) in non-HIV immunocompromised patients. Cochrane Database Syst Rev 2014;26.

15 Humar A, Lebranchu Y, Vincenti F, et al. The efficacy and safety of 200 days valganciclovir cytomegalovirus prophylaxis in high-risk kidney transplant recipients. Am J Transplant 2010;10:1228-37.

16 Liu AW, Jutivorakool K, Fisher CE, et al. Comparison of preemptive therapy and antiviral prophylaxis for prevention of cytomegalovirus in seropositive liver transplant recipients. Transplantation 2018;102:632-9.

17 Winston DJ, Limaye AP, Pelletier S, et al. Randomized, doubleblind trial of anidulafungin versus fluconazole for prophylaxis of invasive fungal infections in high-risk liver transplant recipients. Am J 2014;14:2758-64.

18 van der Beek MT, Berger SP, Vossen ACTM, et al. Preemptive versus sequential prophylactic-preemptive treatment regimens for cytomegalovirus in renal transplantation: comparison of treatment failure and antiviral resistance. Transplantation 2010;89:320-6.

19 Huang X, Chen $\mathrm{H}$, Han M, et al. Multicenter, randomized, openlabel study comparing the efficacy and safety of micafungin versus itraconazole for prophylaxis of invasive fungal infections in patients undergoing hematopoietic stem cell transplant. Biol Blood Marrow Transplant 2012;18:1509-16.

20 Helfrich $\mathrm{M}$, Dorschner $\mathrm{P}$, Thomas $\mathrm{K}$, et al. A retrospective study to describe the epidemiology and outcomes of opportunistic infections after abdominal organ transplantation. Transpl Infect Dis 2017:19:e12691.

21 Liberati Aet al. The PRISMA statement for reporting systematic reviews and meta-analyses of studies that evaluate health care interventions: explanation and elaboration. Ann Intern Med 2009;151:W-W-94.

22 Higgins JPT, Sterne JAC, Savović J, et al. A revised tool for assessing risk of bias in randomized trials in: Chandler $\mathrm{J}$, McKenzie $\mathrm{J}$, Boutron I, Welch V (editors). Cochrane methods. Cochrane Database Syst Rev 2016;10.

23 Sterne JAC, Hernán MA, Reeves BC, et al. ROBINS-I: a tool for assessing risk of bias in non-randomised studies of interventions. BMJ 2016;355.

24 Humar A, Limaye AP, Blumberg EA, et al. Extended valganciclovir prophylaxis in D+/R- kidney transplant recipients is associated with long-term reduction in cytomegalovirus disease: two-year results of the impact study. Transplantation 2010;90:1427-31.
25 Padullés A, Colom H, Bestard O, et al. Contribution of population pharmacokinetics to dose optimization of ganciclovir-valganciclovir in solid-organ transplant patients. Antimicrob Agents Chemother 2016:60:1992-2002.

26 Finlen Copeland CA, Davis WA, Snyder LD, et al. Long-Term efficacy and safety of 12 months of valganciclovir prophylaxis compared with 3 months after lung transplantation: a single-center, long-term follow-up analysis from a randomized, controlled cytomegalovirus prevention trial. J Heart Lung Transplant 2011;30:990-6.

27 Palmer S, Limaye A, Banks M, et al. Extended valganciclovir prophylaxis to prevent cytomegalovirus after lung transplantation: a randomized, controlled trial. Ann Intern Med 2010;152:761-9.

28 Chawla JS, Ghobadi A, Mosley J, et al. Oral valganciclovir versus ganciclovir as delayed pre-emptive therapy for patients after allogeneic hematopoietic stem cell transplant: a pilot trial (04-0274) and review of the literature. Transpl Infect Dis 2012;14:259-67.

29 Boeckh M, Nichols WG, Chemaly RF, et al. Valganciclovir for the prevention of complications of late cytomegalovirus infection after allogeneic hematopoietic cell transplantation. Ann Intern Med 2015;162:1-10.

$30 \mathrm{Kim}$ ST, Lee MH, Kim SY, et al. A randomized trial of preemptive therapy for prevention of cytomegalovirus disease after allogeneic hematopoietic stem cell transplantation. Int J Hematol 2010;91:886-91.

31 Halim M, Al-Otaibi T, Gheith O, et al. Efficacy and safety of lowdose versus standard-dose valganciclovir for prevention of cytomegalovirus disease in intermediate-risk kidney transplant recipients. Exp Clin Transplant 2016;14:526-34.

32 Gheith O, Halim MA, Al-Otaibi T, et al. Successful cost-effective prevention of cytomegalovirus disease in kidney transplant recipients using low-dose valganciclovir. Exp Clin Transplant 2017;15:156-63.

33 Witzke O, Hauser IA, Bartels M, et al. Valganciclovir prophylaxis versus preemptive therapy in Cytomegalovirus-Positive renal allograft recipients: 1 -year results of a randomized clinical trial. Transplantation 2012;93:61-8.

34 Witzke O, Nitschke M, Bartels M, et al. Valganciclovir Prophylaxis Versus Preemptive Therapy in Cytomegalovirus-Positive Renal Allograft Recipients: long-term Results after 7 Years of a Randomized Clinical Trial. Transplantation. 2018;102(5):876-882. doi

35 Reischig T, Hribova P, Jindra P, et al. Long-Term outcomes of pre-emptive valganciclovir compared with valacyclovir prophylaxis for prevention of cytomegalovirus in renal transplantation. JASN 2012;23:1588-97.

36 Reischig T, Kacer M, Jindra P, et al. Randomized trial of valganciclovir versus valacyclovir prophylaxis for prevention of cytomegalovirus in renal transplantation. CJASN 2015;10:294-304.

37 Reischig T, Prucha M, Sedlackova L, et al. Valganciclovir prophylaxis against cytomegalovirus impairs lymphocyte proliferation and activation in renal transplant recipients. Antivir Ther 2011;16:1227-35.

38 Togashi J, Sugawara Y, Hashimoto M, et al. Oral valganciclovir versus intravenous ganciclovir as preemptive treatment for cytomegalovirus infection after living donor liver transplantation: a randomized trial. Biosci Trends 2011:5:217-22.

39 Montejo M, Porto M, Carmon O, et al. Prophylactic therapy with valganciclovir in high-risk (cytomegalovirus $D+/ R$-) kidney transplant recipients: a single-center experience. Proceedings 2010;42:2947-9.

40 Nanmoku K, Shinzato T, Kubo T, et al. Prevention of late-onset cytomegalovirus infection and disease in Donor-Positive/RecipientNegative kidney transplant recipients using low-dose valganciclovir. Transplant Proc 2018;50:124-9.

41 Perrottet N, Csajka C, Pascual M, et al. Population pharmacokinetics of ganciclovir in solid-organ transplant recipients receiving oral valganciclovir. Antimicrob Agents Chemother 2009;53:3017-23.

42 McGillicuddy JW, Weimert NA, Taber DJ, et al. Can preemptive cytomegalovirus monitoring be as effective as universal prophylaxis when implemented as the standard of care in patients at moderate risk? Transplantation 2010;89:1218-23.

43 Mitsani D, Nguyen MH, Shields RK, et al. Prospective, observational study of voriconazole therapeutic drug monitoring among lung transplant recipients receiving prophylaxis: factors impacting levels of and associations between serum troughs, efficacy, and toxicity. Antimicrob Agents Chemother 2012;56:2371-7.

44 Cordonnier C, Rovira M, Maertens J, et al. Voriconazole for secondary prophylaxis of invasive fungal infections in allogeneic stem cell transplant recipients: results of the VOSIFI study. Haematologica 2010;95:1762-8.

45 Takenaka K, Nagafuji K, Takase K, et al. Initial low-dose valganciclovir as a preemptive therapy is effective for cytomegalovirus infection in allogeneic hematopoietic stem cell transplant recipients. Int $J$ Hematol 2012:96:94-100. 
46 Park S-Y, Lee S-O, Choi S-H, et al. Efficacy and safety of lowdose ganciclovir preemptive therapy in allogeneic haematopoietic stem cell transplant recipients compared with conventional-dose ganciclovir: a prospective observational study. J Antimicrob Chemother 2012;67:1486-92.

47 Perrella A, Esposito C, Pisaniello D, et al. Role of liposomal amphotericin B prophylaxis after liver transplantation compared with fluconazole for high-risk patients. impact on infections and mortality within one year. Transplant Proc 2012;44:1977-81.

48 Chaftari A-M, Hachem RY, Ramos E, et al. Comparison of posaconazole versus Weekly amphotericin B lipid complex for the prevention of invasive fungal infections in hematopoietic stem-cell transplantation. Transplantation J 2012;94:302-8.

49 Park S, Kim K, Jang JH, et al. Randomized trial of micafungin versus fluconazole as prophylaxis against invasive fungal infections in hematopoietic stem cell transplant recipients. $J$ Infect 2016;73:496-505.

50 Manuel O, Kralidis G, Mueller NJ, et al. Impact of antiviral preventive strategies on the incidence and outcomes of cytomegalovirus disease in solid organ transplant recipients. Am J Transplant 2013;13:2402-10.10.1111/ajt.12388

51 Hazell L, Shakir SAW. Under-Reporting of adverse drug reactions. Drug Safety 2006;29:385-96.

52 Golder S, Loke YK, Wright K, et al. Reporting of adverse events in published and unpublished studies of health care interventions: a systematic review. PLoS Med 2016;13:e1002127.

53 Chen K, Cheng MP, Hammond SP, et al. Antiviral prophylaxis for cytomegalovirus infection in allogeneic hematopoietic cell transplantation. Blood Adv 2018;2:2159-75.

54 Gagelmann N, Ljungman P, Styczynski J, et al. Comparative efficacy and safety of different antiviral agents for cytomegalovirus prophylaxis in allogeneic hematopoietic cell transplantation: a systematic review and meta-analysis. Biol Blood Marrow Transplant 2018.

55 You B, Maillet D, Chen EX, et al. Quality of reporting of modern randomized controlled trials in medical oncology: a systematic review. JNCl J Natl Cancer Inst 2012;104:982-9.

56 Huwiler-Müntener K, Juni P, Junker C. Quality of reporting of randomized trials as a measure of methodologic quality. JAMA 2002;287:2801-4.

57 Alley AB, Seo J-W, Hong S-T. Reporting results of research involving human subjects: an ethical obligation. J Korean Med Sci 2015;30:673-5

58 Schulz KF, Altman DG. Statement: updated guidelines for reporting parallel group randomised trials. BMJ 2010;2010.

59 Ben Goldacre HD, Powell-Smith A, Dale A, et al. The compare trials project.

60 loannidis JPAet al. Better reporting of harms in randomized trials: an extension of the CONSORT statement. Ann Intern Med 2004:141:781-8.

61 Betts BC, Young J-AH, Ustun C, et al. Human herpesvirus 6 infection after hematopoietic cell transplantation: is routine surveillance necessary? Biology of Blood and Marrow Transplantation 2011:17:1562-8.

62 Cooley L, Dendle C, Wolf J, et al. Consensus guidelines for diagnosis, prophylaxis and management of Pneumocystis jirovecii pneumonia in patients with haematological and solid malignancies, 2014. Intern Med J 2014;44:1350-63.

63 Ivulich S, Westall G, Dooley M, et al. The evolution of lung transplant immunosuppression. Drugs 2018;78:965-82.

64 Krisl JC, Doan VP, Chemotherapy DVP. Chemotherapy and transplantation: the role of immunosuppression in malignancy and a review of antineoplastic agents in solid organ transplant recipients. Am J Transplant 2017;17:1974-91.

65 Söderlund C, Rådegran G. Immunosuppressive therapies after heart transplantation - the balance between under- and overimmunosuppression. Transplant Rev 2015;29:181-9.10.1016/j. trre.2015.02.005

66 Baron D, Giral M, Brouard S. Reconsidering the detection of tolerance to individualize immunosuppression minimization and to improve long-term kidney graft outcomes. Transpl Int 2015;28:938-59.

67 Hand J. Strategies for antimicrobial stewardship in solid organ transplant recipients. Infect Dis Clin North Am 2018;32:535-50.

68 de Vries ST, Denig P, Lasheras Ruiz C, et al. Interest in a mobile APP for two-way risk communication: a survey study among European healthcare professionals and patients. Drug Saf 2018;41:697-712.

69 Vallejo C, Vazquez L, Cabrera Martin JR, et al. Treatment of invasive fungal infections in high-risk haematological patients: what have we learnt in the past 10 years? Rev Esp Quimioter 2013;26:378-86.

70 Freeman RB, Cohen JT. Transplantation Risks and the Real World: What Does 'High Risk' Really Mean? Am J Transplant 2009;9:23-30.

71 Roberts JA, Abdul-Aziz MH, Lipman J, et al. Individualised antibiotic dosing for patients who are critically ill: challenges and potential solutions. Lancet Infect Dis 2014;14:498-509.

72 Larizgoitia I, Bouesseau M-C, Kelley E. Who efforts to promote reporting of adverse events and global learning. $J$ Public health Res 2013;2:29-e29.

73 Razonable RR, Hayden RT. Clinical utility of viral load in management of cytomegalovirus infection after solid organ transplantation. Clin Microbiol Rev 2013;26:703-27.

74 Boch T, Spiess B, Cornely OA, et al. Diagnosis of invasive fungal infections in haematological patients by combined use of galactomannan, 1,3- $\beta$-D-glucan, Aspergillus PCR, multifungal DNAmicroarray, and Aspergillus azole resistance PCRs in blood and bronchoalveolar lavage samples: results of a prospective multicentre study. Clin Microbiol Infect 2016;22:862-8.

75 Patzer RE, Serper M, Reese PP, et al. Medication understanding, non-adherence, and clinical outcomes among adult kidney transplant recipients. Clin Transplant 2016;30:1294-305.

76 Zanetti-Yabur A, Rizzo A, Hayde N, et al. Exploring the usage of a mobile phone application in transplanted patients to encourage medication compliance and education. Am J Surg 2017;214:743-7. 